\section{Water fluoridation safe and effective}

Public Health England (PHE) published a report on Water fluoridation: Health monitoring in England 2018 ${ }^{1}$ in March this year, on behalf of the Secretary of State for Health and Social Care. It is the second such report after the first was published in March 2014. The findings of both reports are consistent with the view that water fluoridation is an effective and safe public health measure.

The report found that:

- Five-year-olds in areas with water fluoridation schemes were much less likely to experience tooth decay, and less likely to experience more severe decay than in areas without schemes

- The chances of having a tooth/teeth removed in hospital because of decay were also much lower in areas with water fluoridation schemes

- Children from all areas benefited from fluoridation, but children from relatively deprived areas benefited the most

- Dental fluorosis, at a level that may affect the appearance of teeth, was observed in $10 \%$ of children/young people examined in two fluoridated cities; however, there was no difference between children and young people surveyed in fluoridated and non-fluoridated cities when asked about their opinion on the appearance of their teeth, taking into account concerns which have resulted from any cause (for example, poor alignment, decay, trauma or fluorosis)

- Taken alongside the existing wider research, the results do not provide convincing evidence of higher rates of hip fracture, Down syndrome, kidney stones, bladder cancer, or osteosarcoma (a cancer of the bone) due to fluoridation schemes.

Professor John Newton, Director of Health Improvement at PHE, said: 'The evidence in this report shows water fluoridation is a safe and effective method to reduce tooth decay, especially among deprived communities. We would encourage local authorities to consider this evidence carefully when deciding on their plans to improve dental health in their areas'.

The Oral Health Foundation referred to the report as 'paving the way to eliminating children's dental health crisis in England. CEO Dr Nigel Carter OBE said: 'The single biggest improvement to the oral health of Britain in recent years came with the introduction of fluoride in toothpastes and that can be eclipsed further with more water fluoridation schemes.

'During my time as a dentist in Birmingham, I witnessed firsthand the huge benefits of water fluoridation, with children from Birmingham, which had water with added fluoride, having little or no decay and their neighbours from then un-fluoridated Sandwell experiencing significantly higher levels of decay. I could literally tell which side of the road they lived on by looking at their teeth.'

1. Public Health England. Water fluoridation: health monitoring report for England 2018. 22 March 2018. Available at: https://www.gov.uk/government/publications/water-fluoridation-health-monitoring-report-for-england-2018 (accessed April 2018).

\section{'Keep Smiling' campaign launched for elderly}

On 19 April the Relatives \& Residents

Association (R\&RA) launched a 'Keep

Smiling' campaign to highlight the impor-

tance of dental health.

At the Association's AGM, R\&RA Chair

Judy Downey emphasised what a crucial area

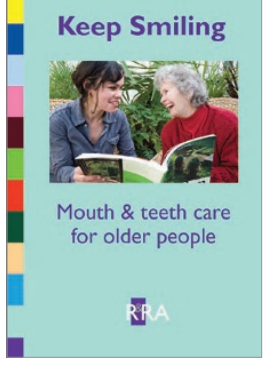

of care dental health is, and that it matters not only for good health, but for all aspects of well-being and self-esteem: 'Older people today are more likely to keep their natural teeth than ever before. They have higher expectations of a healthy mouth and looking good.

'As the handbook points out, it's hard to keep smiling when you've got toothache or a problem with your mouth. As a person's ability to care for their teeth and dentures gets harder so does the general health of their mouth unless they have the support of those who care for them.

The 'Keep Smiling' campaign builds on the R\&RA's successful Keys to Care programme, supporting care workers in their day-today work. The publication is available to download at: http://www. relres.org/wp-content/uploads/Keep-Smiling.pdf. It explains and shows care workers and carers useful skills, tools and techniques, as well as dental care in action in different care settings. There is an associated video in production. 